\title{
THE MASSIVE PROGENITOR OF THE POSSIBLE TYPE II-LINEAR SUPERNOVA 2009hd IN MESSIER 66
}

\author{
Nancy Elias-Rosa ${ }^{1,2,3}$, Schuyler D. Van DyK ${ }^{1}$, Weidong Li ${ }^{2}$, JefFrey M. Silverman $^{2}$, RyAn J. Foley $^{4,12}$, \\ Mohan Ganeshalingam ${ }^{2}$, Jon C. Mauerhan ${ }^{1}$, Erki Kankare ${ }^{5,6}$, Saurabh Jha ${ }^{7}$, Alexei V. Filippenko ${ }^{2}$, \\ John E. Beckman ${ }^{8,9}$, Edo Berger ${ }^{4}$, Jean-Charles Cuillandre ${ }^{10}$, and Nathan Smith ${ }^{2,11}$ \\ ${ }^{1}$ Spitzer Science Center, California Institute of Technology, Pasadena, CA 91125, USA; nelias@ ice.csic.es \\ ${ }^{2}$ Department of Astronomy, University of California, Berkeley, CA 94720-3411, USA \\ ${ }^{3}$ Institut de Ciències de lÕEspa'i (IEEC-CSIC), Facultat de Ciències, Campus UAB, 08193 Bellaterra, Spain \\ ${ }^{4}$ Harvard/Smithsonian Center for Astrophysics, Cambridge, MA 02138, USA \\ ${ }^{5}$ Tuorla Observatory, Department of Physics and Astronomy, University of Turku, 21500 Piikkiö, Finland \\ ${ }^{6}$ Nordic Optical Telescope, Apartado 474, E-38700 Santa Cruz de La Palma, Spain \\ ${ }^{7}$ Department of Physics and Astronomy, Rutgers, The State University of New Jersey, Piscataway, NJ 08854, USA \\ ${ }^{8}$ Instituto de Astrofisica de Canarias, E-38200 La Laguna, Tenerife, Spain \\ ${ }^{9}$ Consejo Superior de Investigaciones Científicas, Madrid, Spain \\ ${ }^{10}$ Canada-France-Hawaii Telescope Corporation, Kamuela, HI 96743, USA \\ ${ }^{11}$ Steward Observatory, University of Arizona, Tucson, AZ 85721-0065, USA \\ Received 2010 November 9; accepted 2011 August 12; published 2011 October 28
}

\begin{abstract}
We present early- and late-time photometric and spectroscopic observations of supernova (SN) 2009hd in the nearby spiral galaxy NGC 3627 (M66). This SN is one of the closest to us in recent years and provides an uncommon opportunity to observe and study the nature of SNe. However, the object was heavily obscured by dust, rendering it unusually faint in the optical given its proximity. We find that the observed properties of SN 2009hd support its classification as a possible Type II-Linear SN (SN II-L), a relatively rare subclass of core-collapse SNe. High-precision relative astrometry has been employed to attempt to identify an SN progenitor candidate, based on a pixel-by-pixel comparison between Hubble Space Telescope (HST) F555W and F814W images of the SN site prior to explosion and at late times. A progenitor candidate is identified in the F814W images only; this object is undetected in F555W. Significant uncertainty exists in the astrometry, such that we cannot definitively identify this object as the SN progenitor. Via insertion of artificial stars into the pre-SN HST images, we are able to constrain the progenitor's properties to those of a possible supergiant, with intrinsic absolute magnitude $M_{\mathrm{F} 555 \mathrm{~W}}^{0} \gtrsim-7.6 \mathrm{mag}$ and intrinsic color $(V-I)^{0} \gtrsim 0.99 \mathrm{mag}$. The magnitude and color limits are consistent with a luminous red supergiant (RSG); however, they also allow for the possibility that the star could have been more yellow than red. From a comparison with theoretical massive-star evolutionary tracks which include rotation and pulsationally enhanced mass loss, we can place a conservative upper limit on the initial mass for the progenitor of $M_{\text {ini }} \lesssim 20 M_{\odot}$. If the actual mass of the progenitor is near the upper range allowed by our derived mass limit, then it would be consistent with that for the identified progenitors of the SN II-L 2009kr and the high-luminosity SN II-Plateau (II-P) $2008 \mathrm{cn}$. The progenitors of these three SNe may possibly bridge the gap between lower-mass RSGs that explode as SNe II-P and luminous blue variables, or more extreme RSGs, from which the more exotic SNe II-narrow may arise. Very late time imaging of the SN 2009hd site may provide us with more clues regarding the true nature of its progenitor.
\end{abstract}

Key words: galaxies: individual (NGC 3627) - stars: evolution - supernovae: general - supernovae: individual (SN 2009hd)

\section{INTRODUCTION}

The Type II-Linear supernovae (SNe II-L) are among the least common and, therefore, most poorly studied subclasses of corecollapse supernovae (CC-SNe). They represent $6.4 \%-10 \%$ of all CC-SNe (Smith et al. 2011; Li et al. 2011). The spectra of $\mathrm{SNe}$ II-L are similar to those of the much more common $\mathrm{SNe}$ II-Plateau (II-P), but SNe II-L are distinguished by the shapes of their light curves. While light curves of SNe II-P exhibit a relatively constant plateau for $\sim 100 \mathrm{~d}$ after an initial peak in the first few days, the light curves of SNe II-L instead show a linear decline commencing just after the initial peak (e.g., Barbon et al. 1979). The very short, or nonexistent, plateau phase for SNe II-L may well result from a low-mass $\mathrm{H}$ envelope at the time of explosion (between 1 and $5 M_{\odot}$; Swartz et al. 1991). The envelope can be further reduced through pre-SN mass loss, and if so, we would infer that the mass of the progenitor star is higher

\footnotetext{
${ }^{12}$ Clay Fellow.
}

than that expected for SNe II-P, $8.5_{-1.5}^{+1.0} \lesssim M_{\text {ini }} \lesssim 16.5 \pm 1.5 M_{\odot}$ (Smartt et al. 2009). The direct identification of the progenitor of the SN II-L 2009kr (Elias-Rosa et al. 2010) provides evidence that this indeed may be the case (note that Fraser et al. 2010 suggest that this SN is a spectrally peculiar SN II-P). Clearly, the identification of the progenitors of other SNe II-L is necessary to establish a trend, if one exists.

In this paper, we present the case of SN 2009hd $(\alpha=$ $11^{\mathrm{h}} 20^{\mathrm{m}} 16^{\mathrm{s}} .90, \delta=+12^{\circ} 58^{\prime} 47^{\prime \prime} .1$; J2000.0) in NGC 3627 (Messier 66, M66). This SN was discovered by Monard (2009) on 2009 July 2.69 (UT dates are used throughout) and was spectroscopically classified as a reddened SN II (Kasliwal et al. 2009) with slow evolution (Kasliwal 2009), and, subsequently, as an SN II-P (Berger et al. 2009). Maoz (2009) first identified a possible progenitor star in high spatial resolution archival Hubble Space Telescope (HST) images obtained between 1997 and 1998. Here, we show that both the photometric and spectroscopic observations suggest that this object is, in fact, an SN II-L. We also present a substantially improved identification of 


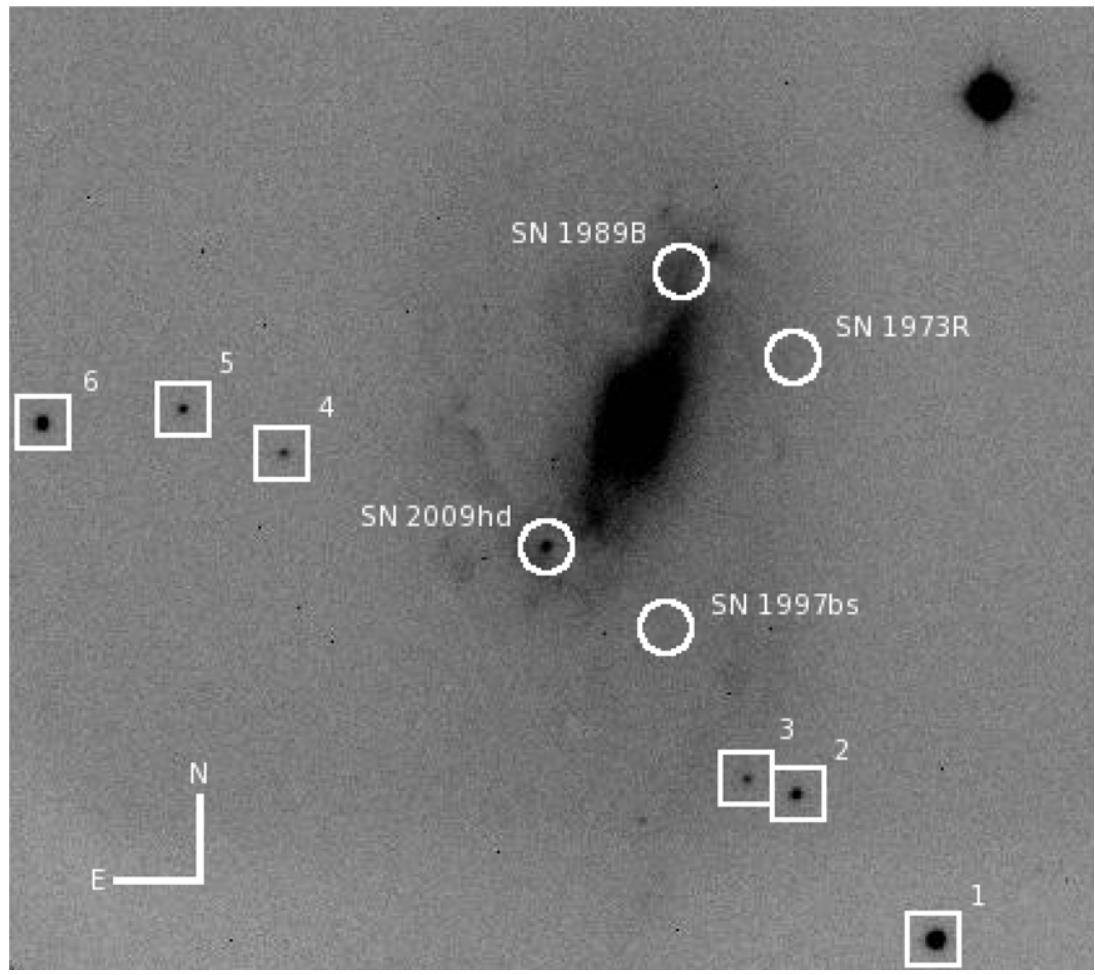

Figure 1. I-band image of SN 2009hd in NGC 3627 (M66) obtained with the $1.3 \mathrm{~m}$ SMARTS telescope+ANDICAM at CTIO on 2009 July 10 (field of view $\sim 6^{\prime} \times 6^{\prime}$ ). The sites of SNe 1973R, 1989B, and 1997bs, as well as the local photometric sequence stars, are indicated.

the progenitor, based on a pixel-by-pixel comparison between the pre-SN HST data and late-time HST images of the SN. We note that M66 has been host to two other known SNe, the SN II 1973R (Ciatti \& Rosino 1977) and the Type Ia SN 1989B (Wells et al. 1994), as well as the "SN impostor" SN 1997bs (Van Dyk et al. 2000); see Figure 1.

\section{THE NATURE OF SN 2009hd}

\subsection{Photometry}

Optical (BVRI) images of SN 2009hd were obtained with the $1.3 \mathrm{~m}$ SMARTS+ANDICAM at Cerro Tololo Inter-American Observatory (CTIO), the Palomar $1.5 \mathrm{~m}$ telescope, the $2.0 \mathrm{~m}$ Liverpool Telescope and the $2.6 \mathrm{~m}$ Nordic Optical Telescope (NOT) at the Roque de los Muchachos Observatory, the $6.5 \mathrm{~m}$ Baade Magellan telescope, and the $10 \mathrm{~m}$ Keck II telescope. The photometric observations were processed using IRAF ${ }^{13}$ routines with the standard recipe for CCD images (trimming, overscan, bias, and flat-field corrections). Due to the location of SN 2009hd along a bright spiral arm of M66, contamination of the photometry by the host-galaxy light was a serious problem. We therefore used the template-subtraction technique to remove this background and hence to measure the $\mathrm{SN}$ magnitudes more accurately. The template images of M66 were obtained with the Kitt Peak National Observatory $2.1 \mathrm{~m}$ telescope, under 1" seeing conditions, about seven years prior to the SN discovery (Kennicutt et al. 2003). The instrumental magnitudes of the SN and the reference stars in the SN field were measured in the subtracted images using the point-spread function (PSF) fitting

13 IRAF (Image Reduction and Analysis Facility) is distributed by the National Optical Astronomy Observatory, which is operated by the Association of Universities for Research in Astronomy, Inc. (AURA), under cooperative agreement with the National Science Foundation (NSF).
Table 1

Adopted Magnitudes for the Local Sequence Stars in Figure 1

\begin{tabular}{lcccc}
\hline \hline Star & $\begin{array}{c}B \\
(\mathrm{mag})\end{array}$ & $\begin{array}{c}V \\
(\mathrm{mag})\end{array}$ & $\begin{array}{c}R \\
(\mathrm{mag})\end{array}$ & $\begin{array}{c}I \\
(\mathrm{mag})\end{array}$ \\
\hline 1 & $13.72 \pm 0.01$ & $13.17 \pm 0.01$ & $12.83 \pm 0.01$ & $12.50 \pm 0.01$ \\
2 & $16.26 \pm 0.01$ & $15.47 \pm 0.01$ & $14.97 \pm 0.01$ & $14.49 \pm 0.03$ \\
3 & $17.16 \pm 0.01$ & $16.54 \pm 0.01$ & $16.16 \pm 0.02$ & $15.77 \pm 0.01$ \\
4 & $16.77 \pm 0.01$ & $16.24 \pm 0.01$ & $15.92 \pm 0.01$ & $15.55 \pm 0.01$ \\
5 & $16.56 \pm 0.01$ & $15.95 \pm 0.01$ & $15.59 \pm 0.01$ & $15.18 \pm 0.01$ \\
6 & $18.03 \pm 0.01$ & $16.49 \pm 0.01$ & $15.41 \pm 0.01$ & $13.49 \pm 0.24$
\end{tabular}

technique with the DAOPHOT package (Stetson 1987) within IRAF.

In order to calibrate the instrumental magnitudes to the standard photometric system, we used the magnitudes and colors of the local sequence stars in the SN field (Figure 1 and Table 1). These values were measured from BVRI images of NGC 3627 obtained under photometric conditions on 1999 December 17 with the $1.5 \mathrm{~m}$ telescope at Palomar Observatory (see Van Dyk et al. 2000).

SN 2009hd was observed during the first $\sim 20 \mathrm{~d}$ after discovery, but it subsequently became too close to the Sun in the sky. It was observed again five months later (Table 2). From the discovery dates given by Monard (2009) and Yamaoka \& Itagaki (2009), we constrained the explosion date to be 2009 June $19 \pm 3$, or JD 2,455,002 \pm 3 . From the light curves of a sample of SNe II-L, it takes $\sim 9 \mathrm{~d}$ from explosion to $B$ maximum (Li et al. 2011). The $B V R I$ light curves for SN 2009hd are shown in Figure 2, relative to $B$ maximum (2009 June $28 \pm 3$, or JD $2,455,011 \pm 3$ ). For comparison, we also show the light curves of the SNe II-L 1990K (Cappellaro et al. 1995) and 2009kr (EliasRosa et al. 2010), and the SNe II-P 1999em (Hamuy et al. 2001; Leonard et al. 2002; Elmhamdi et al. 2003) and 1992H 


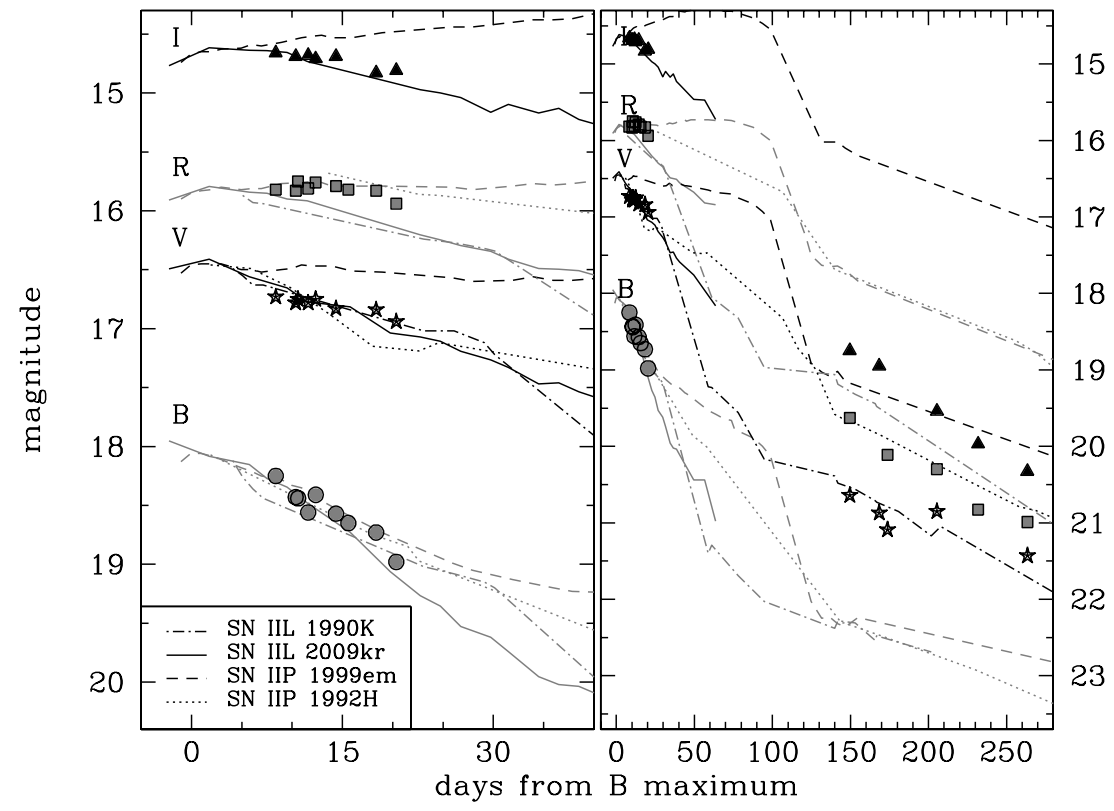

Figure 2. Left: early-time ( $\leqslant 40 \mathrm{~d}$ after $B$-band maximum) optical light curves of SN 2009hd, together with those of the SNe II-L 1990K (dot-dashed lines) and 2009kr (solid lines), and of the SNe II-P 1999em (dashed lines) and 1992H (dotted lines). Right: optical light curves of SN 2009hd, including late-time data. The comparison light curves are adjusted in time and magnitude to match those of SN 2009hd.

Table 2

Photometry of SN 2009hd

\begin{tabular}{lcccccc}
\hline \hline Date & $\begin{array}{c}\text { JD } \\
-2,400,000.00\end{array}$ & $\begin{array}{c}B \\
(\mathrm{mag})\end{array}$ & $\begin{array}{c}V \\
(\mathrm{mag})\end{array}$ & $\begin{array}{c}R \\
(\mathrm{mag})\end{array}$ & $\begin{array}{c}I \\
(\mathrm{mag})\end{array}$ & Source \\
\hline 2009 Jul 6 & 55019.48 & $18.25 \pm 0.05$ & $16.73 \pm 0.06$ & $15.82 \pm 0.05$ & $14.66 \pm 0.04$ & CTIO \\
2009 Jul 8 & 55021.46 & $18.43 \pm 0.04$ & $16.78 \pm 0.03$ & $15.83 \pm 0.04$ & $14.69 \pm 0.04$ & CTIO \\
2009 Jul 9 & 55021.70 & $18.44 \pm 0.06$ & $16.75 \pm 0.03$ & $15.75 \pm 0.04$ & $\ldots$ & P60 \\
2009 Jul 10 & 55022.70 & $18.56 \pm 0.05$ & $16.78 \pm 0.04$ & $15.81 \pm 0.02$ & $14.68 \pm 0.04$ & P60 \\
2009 Jul 10 & 55023.45 & $18.41 \pm 0.07$ & $16.75 \pm 0.02$ & $15.76 \pm 0.03$ & $14.71 \pm 0.03$ & CTIO \\
2009 Jul 12 & 55025.46 & $18.57 \pm 0.08$ & $16.83 \pm 0.06$ & $15.79 \pm 0.06$ & $14.69 \pm 0.04$ & CTIO \\
2009 Jul 14 & 55026.69 & $18.65 \pm 0.03$ & $\ldots$ & $15.82 \pm 0.06$ & $\ldots$ & P60 \\
2009 Jul 16 & 55029.46 & $18.73 \pm 0.07$ & $16.84 \pm 0.02$ & $15.83 \pm 0.05$ & $14.83 \pm 0.03$ & CTIO \\
2009 Jul 18 & 55031.46 & $18.98 \pm 0.06$ & $16.94 \pm 0.02$ & $15.94 \pm 0.05$ & $14.81 \pm 0.03$ & CTIO \\
2009 Nov 24 & 55160.71 & $\ldots$ & $20.64 \pm 0.05$ & $19.63 \pm 0.02$ & $18.75 \pm 0.02$ & LT \\
2009 Dec 14 & 55179.58 & $\ldots$ & $20.87 \pm 0.01$ & $\ldots$ & $18.95 \pm 0.01$ & HST \\
2009 Dec 19 & 55184.86 & $\ldots$ & $21.09 \pm 0.10$ & $20.11 \pm 0.08$ & $\ldots$ & Mag \\
2010 Jan 20 & 55216.55 & $\ldots$ & $20.85 \pm 0.04$ & $20.30 \pm 0.05$ & $19.54 \pm 0.04$ & LT \\
2010 Feb 15 & 55242.93 & $\ldots$ & $\ldots$ & $20.83 \pm 0.05$ & $19.97 \pm 0.07$ & Keck \\
2010 Mar 19 & 55274.54 & $\ldots$ & $21.43 \pm 0.09$ & $20.99 \pm 0.08$ & $20.33 \pm 0.13$ & NOT \\
\hline
\end{tabular}

Notes. CTIO: CTIO 1.3 m SMARTS telescope + ANDICAM; P60: Palomar $1.5 \mathrm{~m}$ telescope + CCD camera; LT: $2.0 \mathrm{~m}$ Liverpool Telescope + RATCam; HST: Hubble Space Telescope + ACS camera; Mag: 6.5 m Baade Magellan telescope + IMACS; Keck: 10 m Keck II telescope + DEIMOS; NOT: 2.6 m Nordic Optical Telescope + ALFOSC.

(Clocchiatti et al. 1996). From the light-curve comparison, it appears that SN 2009hd does not follow a standard SN II-P evolution and does not behave like any SN IIb (e.g., SN 1993J; Barbon et al. 1995, not shown in the figure). Instead, it more closely resembles the SN II-L post-maximum decline. During early epochs, the light curves show a steep, almost linear, behavior. A linear fit to the data for the first $12 \mathrm{~d}$ results in decline rates $\beta_{12}^{B}=0.62 \pm 0.05, \beta_{12}^{V}=0.18 \pm 0.03, \beta_{12}^{R}=0.14 \pm 0.03$, and $\beta_{12}^{I}=0.17 \pm 0.02 \mathrm{mag}(12 \mathrm{~d})^{-1}$ in each band. We note the presence of a "bump" in the $B V R$ light curves at $\sim 10-20 \mathrm{~d}$, which seems to follow the "shoulder" exhibited by SN 1990K (Cappellaro et al. 1995). This light-curve feature appears in the case of several SNe II-L (Patat et al. 1994). After $\sim 100 \mathrm{~d}$ following $B$ maximum, the luminosity decline settles down at a significantly lower rate. This behavior is consistent with complete trapping of the energy release from the radioactive decay of ${ }^{56} \mathrm{Co}$ to ${ }^{56} \mathrm{Fe}$. In the phase range $140-260 \mathrm{~d}$, the decline rates are $\gamma^{V}=0.57 \pm 0.08, \gamma^{R}=1.19 \pm 0.10$, and $\gamma^{I}=1.44 \pm 0.12 \mathrm{mag}(100 \mathrm{~d})^{-1}$, which are similar to those found for SN 1990K (Cappellaro et al. 1995).

Figure 3 shows a comparison between the evolution of the absolute $V$ magnitude, $M_{V}$, of SN 2009hd, compared with the SNe II-L 1980K (Barbon et al. 1982; Buta 1982; Tsvetkov 1983), 1986E (Cappellaro et al. 1990), 1990K (Cappellaro et al. 1995), 1994aj (Benetti et al. 1998), 2001cy (Poznanski et al. 2009), and 2009kr (Elias-Rosa et al. 2010), with the SN IIb 1993J (Barbon et al. 1995), and with the well-studied SNe II-P 1992H (Clocchiatti et al. 1996) and 1999em (Hamuy et al. 2001; Leonard et al. 2002; Elmhamdi et al. 2003). The comparison SNe have been corrected for extinction using published estimates 


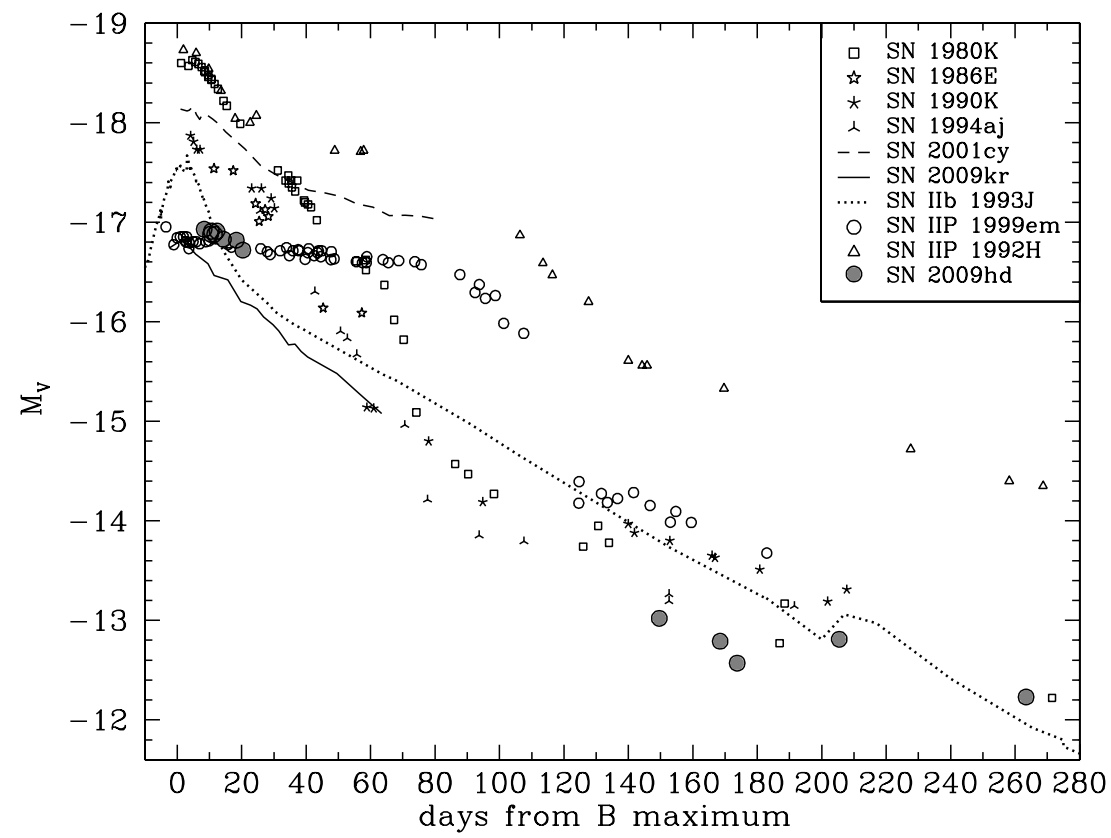

Figure 3. Absolute $V$ light curve of SN 2009hd (filled circles) along with those of the SNe II-L 1980K (empty squares), 1986E (empty stars), 1990K (five-pointed stars), 2001cy (dashed lines), and 2009kr (solid lines); the peculiar SN II-L 1994aj (three-pointed stars); the SN IIb 1993J (dotted lines); and the SNe II-P 1999em (empty circles) and 1992H (empty triangles). Distances and extinction estimates for all SNe, except SN 2009hd, have been adopted from the literature. The magnitudes of SN 2009hd have been corrected for the assumed reddening, $E(B-V)=1.23 \mathrm{mag}$, and the adopted distance modulus, $\mu=29.86 \pm 0.08 \mathrm{mag}$ (Freedman et al. 2001).

and assuming the Cardelli et al. (1989) extinction law. For SN 2009hd, we have adopted a total extinction of $A_{V}=$ $3.80 \pm 0.14 \mathrm{mag}$ (see Section 2.3) and a distance to M66 of $9.4 \mathrm{Mpc}$ (derived from the extinction-corrected Cepheid distance; Freedman et al. 2001). Again, it is possible to see the remarkable similarity between the SNe II-L and SN 2009hd, primarily with SNe $1990 \mathrm{~K}$ and $2009 \mathrm{kr}$, implying that this SN also could be a member of this rare subclass of CC-SNe.

Furthermore, the absolute $(B-V)_{0},(V-R)_{0},(R-I)_{0}$, and $(V-I)_{0}$ color evolution of SN 2009hd are typical of an SN II-L (see Figure 4). The color curves show a rapid evolution from blue to red in the earlier epochs, except for the $(R-I)_{0}$ color curves, which initially exhibit a color decrease for 2-3 d, probably due to the $R$-band "bump." At late times, however, the color becomes significantly more blue than that of other SNe II. Only the $(R-I)_{0}$ color curves for some $\mathrm{SNe} \mathrm{IIb}$, such as SNe 1993J or 2008ax (not shown in the figure; Taubenberger et al. 2011), have a similar behavior at such epochs. This apparent bluer color SN 2009hd is possibly caused by light from neighboring young stars contaminating our late-time measurements when the $\mathrm{SN}$ is faint.

We therefore conclude that SN 2009hd displays the photometric behavior of an SN II-L. We caution, however, that a late plateau may have evaded detection, as a result of the absence of data between $30 \mathrm{~d}$ and $100 \mathrm{~d}$. This could imply that SN 2009hd is actually a hybrid event with characteristics of SNe II-L and SNe II-P, such as SN 1992H (Clocchiatti et al. 1996).

\subsection{Spectroscopy}

Medium-resolution optical spectra of SN 2009hd were obtained on 2009 July 9 with the Magellan/Clay $6.5 \mathrm{~m}$ telescope using the Magellan Echellete (MagE; Marshall et al. 2008) spectrograph, and on 2010 February 15 with the 10 m Keck II telescope using the Deep Imaging Multi-Object Spectrograph (DEIMOS; Faber et al. 2003).

Standard CCD processing and spectrum extraction of the MagE data were accomplished with IRAF routines. The data were extracted using the optimal algorithm of Horne (1986). Low-order polynomial fits to calibration-lamp spectra were used to establish the wavelength scale. Small adjustments derived from night-sky lines in the object frames were applied. For the MagE spectra, the sky was subtracted from the images using the method described by Kelson (2003). We employed IRAF and our own routines to flux calibrate the data and remove telluric lines using the well-exposed continua of spectrophotometric standards (Wade \& Horne 1988; Foley et al. 2003, 2009). CCD processing of the DEIMOS spectra was performed with a modified version of the DEEP pipeline (e.g., Weiner et al. 2005). This produced rectified, sky-subtracted two-dimensional spectra from which one-dimensional spectra were then extracted optimally (Horne 1986). The absolute flux calibration of the spectra was checked against the photometry and, when necessary, the spectra were rescaled. After that, the typical deviation of the spectra from photometry is $<10 \%$ in all bands. We note, however, that the early-time spectrum was observed $\sim 45^{\circ}$ from the parallactic angle (Filippenko 1982) at airmass 2.1, and the flux of the late-time spectrum might be slightly too low because of the presence of a dead column in the CCD next to the trace of the SN; consequently, the shape of the continuum in both spectra could be affected.

A near-infrared (NIR) spectrum was obtained with the Palomar Hale $5 \mathrm{~m}$ telescope using TripleSpec (Herter et al. 2008) on 2009 July 15. After standard CCD processing and before extraction, the contribution of the night-sky lines was removed from one two-dimensional NIR spectral image, subtracting another two-dimensional spectral image with the SN placed in a different position along the slit. In the NIR, the wavelength calibration was performed using the $\mathrm{OH}$ sky lines, 


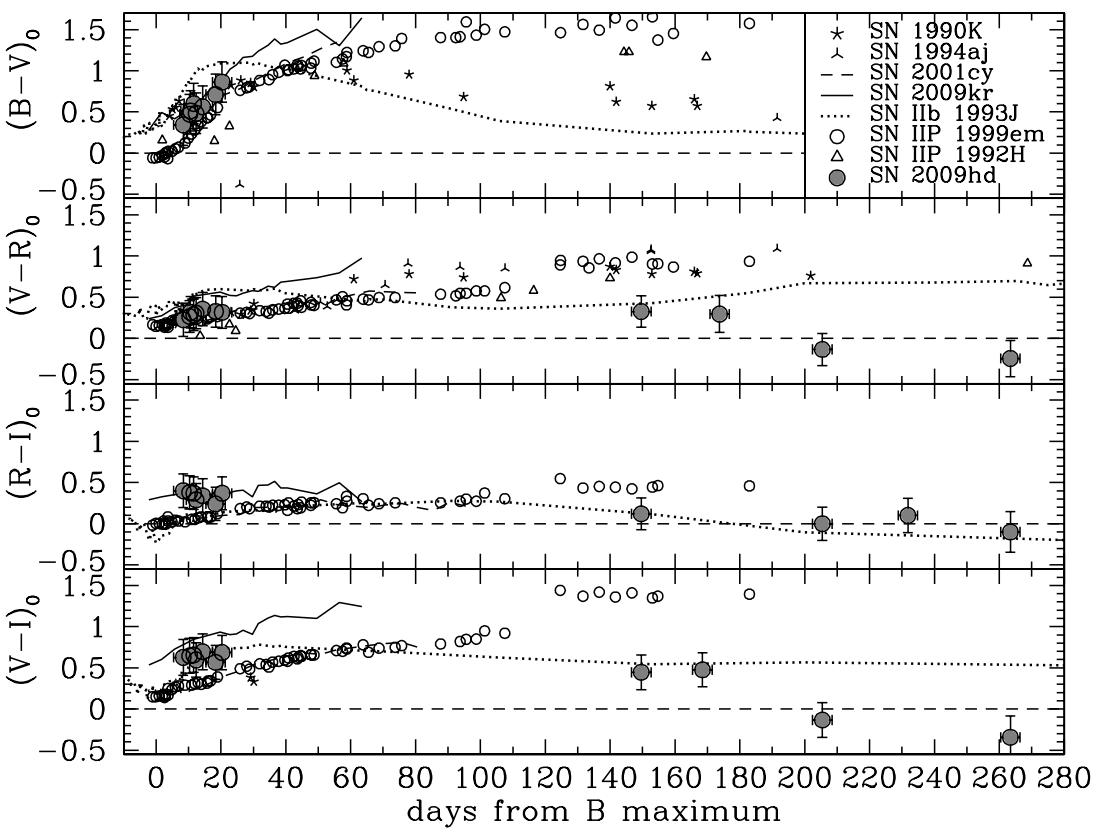

Figure 4. Intrinsic $(B-V)_{0},(V-R)_{0},(R-I)_{0}$, and $(V-I)_{0}$ color curves of SN 2009hd, compared with the intrinsic color evolution of the SNe II-L 1990K (five-pointed stars), 2001cy (dashed lines), and 2009kr (solid lines); the peculiar SN II-L 1994aj (three-pointed stars); the SN IIb 1993J (dotted lines); and the SNe II-P 1999em (empty circles) and 1992H (empty triangles). Distances and extinction estimates for all SNe, except SN 2009hd, have been adopted from the literature. The color of SN 2009hd has been corrected for the assumed reddening, $E(B-V)=1.23 \mathrm{mag}$, and the adopted distance modulus, $\mu=29.86 \pm 0.08 \mathrm{mag}$ (Freedman et al. 2001).

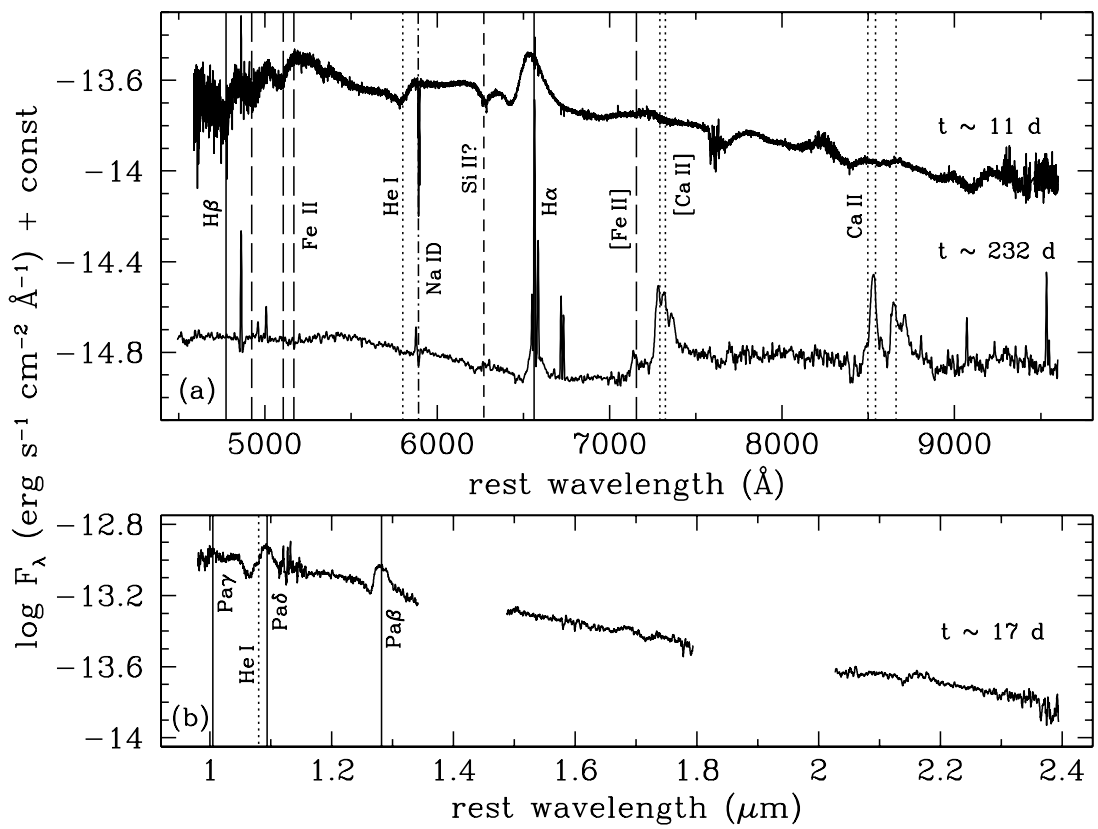

Figure 5. (a) Optical spectral evolution of SN 2009hd. The late-time spectrum appears to have been contaminated by light from stars near the SN. (b) A near-infrared spectrum obtained using TripleSpec with the Palomar $5 \mathrm{~m}$ Hale telescope on 2009 July 15. Spectra in both panels have been corrected for their host-galaxy recession velocities, but not for extinction. Ages are relative to $B$ maximum light. The locations of the most prominent spectral features are marked.

and a telluric absorption correction was derived from observations of the A0V star HR 6944 and applied to the SN data using the IDL package xtellcor (Vacca et al. 2003).

Figure 5 shows the final optical and NIR spectra of SN 2009hd. The most notable feature of the early optical spectrum (Figure 5, top panel) is the very red continuum, due to the appreciable extinction of this SN (see Section 2.3). The spectrum also exhibits relatively weak spectral features, mainly due to metals, and a broad $\mathrm{H} \alpha$ emission component. The absence of a standard P-Cygni absorption component of $\mathrm{H} \alpha$ has also been observed in some other SNe II-L, such as SNe 1979C and $1980 \mathrm{~K}$, and this has been postulated as a characteristic that distinguishes spectroscopically the SNe II-L from the SNe II-P (Schlegel 1996; Filippenko 1997).

An intriguing feature is present on the blue side of $\mathrm{H} \alpha$, at about $6300 \AA$. Similar absorption is also visible in early-time spectra of SNe 1999em (Baron et al. 2000; Dessart \& Hillier 2005) and 2005cs (Pastorello et al. 2006). This feature has been 


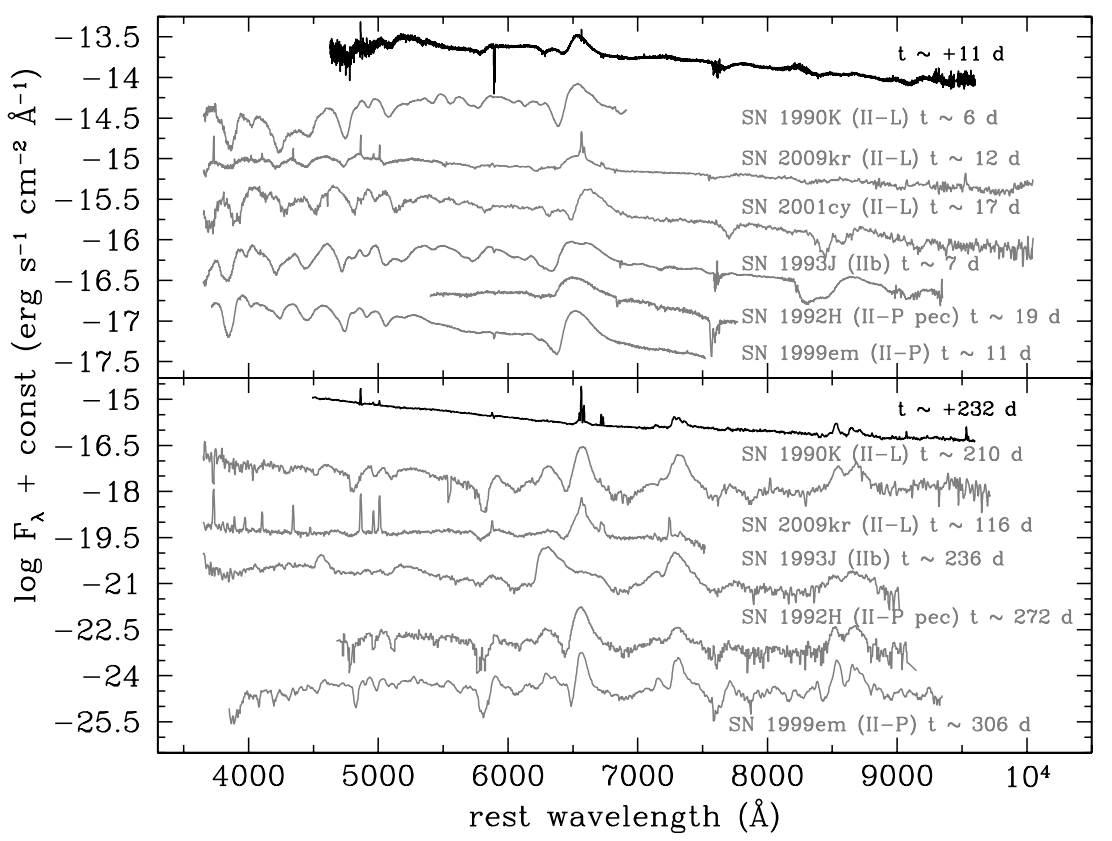

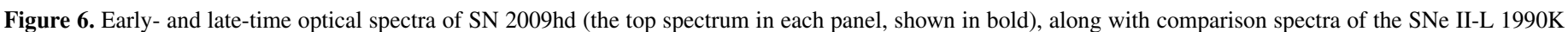

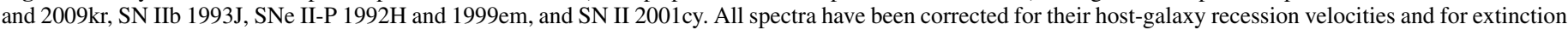
(see the text). Ages are relative to $B$ maximum light.

interpreted either as a high-velocity $\mathrm{H}$ I line, possibly due to an outer H-rich shell, or as Si II $\lambda 6355$ absorption. Unfortunately, the lack of spectra at early times does not allow us to definitively determine which of these explanations is correct. However, since the expansion velocities of the ejecta $\left(\sim 6400\right.$ and $3600 \mathrm{~km} \mathrm{~s}^{-1}$, respectively) for the $\mathrm{H} \alpha$ and the putative $\mathrm{Si}$ II features for $\mathrm{SN}$ 2009hd are similar as those for SN 2005cs at approximately the same epoch (see Figure 6 of Pastorello et al. 2006), we surmise that the absorption near $6300 \AA$ could be more likely due to Si II, rather than to high-velocity hydrogen.

In the late-time spectrum, the continuum has become much bluer than in the early-time spectrum, quite probably as a result of contamination by nearby, young stars, which would have been included within the spectrograph slit, given the seeing conditions. The spectrum shows weaker $\mathrm{H} \alpha$, [Fe II] $\lambda 7155, \mathrm{Ca}$ II $\lambda \lambda 7291,7324$, and [Ca II] $\lambda \lambda 8498,8542,8662$ emission lines (see Figure 5). Since the SN is very close to an HiI region, which was also included in the slit, narrow emission lines of $\mathrm{H} \alpha, \mathrm{H} \beta,[\mathrm{O}$ III] $\lambda 5007,[\mathrm{~N}$ II] $\lambda \lambda 6548,6584$, and [S II] $\lambda \lambda 6717$, 6731 appear superposed on the spectrum.

Finally, the spectrum in the bottom panel of Figure 5 may well be the first-ever NIR spectrum obtained for an SN II-L. We identified lines by comparing the spectrum to that of $\mathrm{SNe}$ II-P such as SN 2002hh (Pozzo et al. 2006). The SN 2009hd spectrum is dominated by lines of the hydrogen Paschen series. The wavelength regions $\sim 1.35-1.50 \mu \mathrm{m}$ and $\sim 1.80-2.00 \mu \mathrm{m}$ were compromised, however, by strong telluric absorption. The relatively featureless nature of this spectrum is reminiscent of the early-time NIR spectra of SN 1993J (Matthews et al. 2002).

The SN 2009hd optical spectra are shown in Figure 6, relative to the spectra at similar epochs of the SNe II-L 1990K (Cappellaro et al. 1995) and 2009kr (Elias-Rosa et al. 2010), as well as of the SN IIb 1993J (Barbon et al. 1995), the SNe II-P 1992H (Clocchiatti et al. 1996) and 1999em (Leonard et al. 2002), and the SN II 2001cy (Poznanski et al. 2009). All of the spectra are shown relative to $B$ maximum; they have been corrected for extinction using published estimates and deredshifted using values from NED. ${ }^{14}$ At all times, SN 2009hd clearly differs from SNe 1993J, 1992H, and 1999em, and more closely resembles SNe $2001 \mathrm{cy}$ and $2009 \mathrm{kr}$, which also exhibit relatively weak lines (such as the weak $\mathrm{H} \alpha$ absorption trough). SN 1990K, also an SN II-L, shows overall stronger features than SN 2009hd. At late times, the features present for SN 2009hd are similar to those of normal SNe II-P, except that they are weaker or nearly absent. The fact that the continuum is dominated by relatively narrow $\mathrm{Ca}$ II and [Ca II] emission may imply the obscuration of the SN progenitor by dust, as well as the destruction of dust in the circumstellar medium (CSM); see, for example, the case of SN 2008S (Prieto et al. 2008; Smith et al. 2009). We also compared SN 2009hd with the prototypical Type II-narrow (IIn) SN $1988 Z$ (not shown in the figure); they appear similar blueward of $\mathrm{H} \alpha$, but quite different on the redward side. Thus, once again we emphasize the resemblance of SN 2009hd to other SNe II-L.

\subsection{Extinction}

To derive consistent estimates of the extinction, $A_{V}$, toward SN 2009hd, we adopted two different methods, all based on the comparison of the SN's spectral energy distribution (SED) and luminosity with those of other "standard" CC-SNe. The estimates we derive below relate to the $\mathrm{SN}$ at early times. There is some hint, from both the color curves and spectrum, that the extinction decreased at late times; however, as discussed above, what we consider the more likely explanation is contamination of the SN light by neighboring stars.

1. We matched simultaneously the absolute $(B-V)_{0}$, $(V-R)_{0},(R-I)_{0}$, and $(V-I)_{0}$ colors curves of SN 2009hd with those of other SNe II-L (see Figure 4). The best match is for $A_{V}=3.9 \pm 0.1 \mathrm{mag}$. This comparison was primarily weighted by the larger number of data points at early epochs.

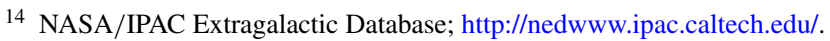


2. The observed extinction was estimated by comparing the early-time optical SED of SN 2009hd with those of SNe $1990 \mathrm{~K}, 2001 \mathrm{cy}$, and $2009 \mathrm{kr}$ at similar epochs. Spectra of the comparison $\mathrm{SNe}$ were first corrected for redshift and extinction, and scaled to the distance of SN 2009hd. The average of the good matches in all cases is $A_{V}=$ $3.7 \pm 0.2 \mathrm{mag}$.

We find that these extinction estimates from the two quite similar methods agree reasonably well with each other. Hence, we adopt $A_{V}^{\text {tot }}=3.80 \pm 0.14$ mag as the extinction toward SN 2009hd. The foreground Galactic component to this extinction is a relatively low $0.11 \mathrm{mag}$ (Schlegel et al. 1998), so, therefore, most of the extinction must be local to the SN.

This extinction estimate is consistent with the constraint on the reddening we can derive from the Magellan + MagE spectrum. We measured the equivalent width (EW) of the Na I D line at the host-galaxy redshift $\left(z_{0}=0.0024\right)$ and found for the two line components, EW(Na I D1 $\lambda 5890)=1.25 \pm 0.05 \AA$ and $\mathrm{EW}(\mathrm{Na}$ I D2 $\lambda 5896)=1.10 \pm 0.05 \AA$. Considering that $\mathrm{EW}(\mathrm{NaI} \mathrm{D} 1)_{\mathrm{hr}}+\mathrm{EW}(\mathrm{NaI} \mathrm{D} 2)_{\mathrm{hr}}=\mathrm{EW}(\mathrm{NaI} \text { D) })_{\mathrm{lr}}$ (EliasRosa 2007), where "hr" and "lr" denote "high" and "low resolution," respectively, this results in EW(Na I D) $=2.35 \pm 0.07$. However, since the ratio between the $\mathrm{Na}$ I $\mathrm{D}$ component lines is close to saturation (1.14, with saturation at 1.1; Spitzer 1948), and given the considerable scatter in the distribution of $\mathrm{SNe}$ in the EW(Na I D) versus $E(B-V)$ plane (e.g., see Elias et al. $2007^{15}$ or Poznanski et al. 2011) and the large variance in this relation found in the literature, the estimated value of $E(B-V)_{\text {tot }}$ can only be considered as a lower limit. For example, using the relation between reddening and $\mathrm{EW}(\mathrm{Na}$ I $\mathrm{D})$ (for all type of $\mathrm{SNe})$ from Elias et al. $(2007, E(B-V)=0.11 \times \mathrm{EW}(\mathrm{Na}$ I D) -0.01 ), and assuming the Cardelli et al. (1989) reddening law, with updated bandpass wavelengths, and a Galactic foreground $E(B-V)=0.03 \mathrm{mag}$ (Schlegel et al. 1998), we estimate $E(B-V)_{\text {tot }} \gtrsim 0.29 \pm 0.03 \mathrm{mag}\left(A_{V} \gtrsim 0.90 \pm 0.09 \mathrm{mag}\right.$ and $\left.E[V-I]_{\text {tot }} \gtrsim 0.35 \pm 0.06 \mathrm{mag}\right)$. If instead we use the relation given by Olivares et al. (2010) for SNe II-P $(E(B-V) \approx 0.25 \times$ $\mathrm{EW}(\mathrm{Na}$ I D) $)$, we estimate $E(B-V)_{\text {tot }} \gtrsim 0.62 \mathrm{mag}$. In both cases, these lower limits are consistent with the two direct extinction estimates above.

We note here the possible temporal evolution of the $\mathrm{Na}$ feature. While it is strong at early times, indicative of the high extinction toward the SN, the feature's strength appears to be reduced by $\sim 20 \%(\mathrm{EW}[\mathrm{Na}$ I D $]=1.87 \pm 0.05)$ at late times. Such variability in the Na I D lines has been observed before-for example, in the faint transient SN 2008S in NGC 6946 (Smith et al. 2009; Botticella et al. 2009), or in thermonuclear SNe, such as SN 2006dd (Stritzinger et al. 2011), and SN 1999cl and SN 2006X (see Blondin et al. 2009 and references therein). However, in these latter two cases, the EW of the lines increased with time, rather than decreased. Considering the expected recombination of $\mathrm{Na}$ with time, such a variation for SN 2009hd, if real, may be due to ionization changes in the CSM, as a result of the weaker radiation field from the $\mathrm{SN}$ at later times. This effect could also be related to the possible decline in the local extinction mentioned in Section 2.2. On the other hand, another, possibly more likely, explanation for the apparent change in the $\mathrm{Na}$ I D EW is dilution of this spectral feature by light from nearby stars and $\mathrm{H}$ II regions, as previously noted.

\footnotetext{
15 http://pos.sissa.it/cgi-bin/reader/conf.cgi? $\operatorname{confid}=60$.
}

\section{IDENTIFICATION OF THE PROGENITOR CANDIDATE}

Shortly after the discovery of SN 2009hd, we isolated 12 archival $^{16} H S T /$ WFPC2 CR-SPLIT image pairs of M66 in F555W $(\sim V)$ and 5 pairs in F814W $(\sim I)$, in order to identify any stars in the images that could be candidates for the SN progenitor. These images were obtained between 1997 November and 1998 January by program GO-6549 (PI: A. Sandage) and used originally to derive a Cepheid-based distance to M66 (Saha et al. 1999). The exposure times in each band were 2400-2500 s. Combining the images in each band over all epochs of the Cepheid observations yields a total image depth of $\sim 16 \mathrm{hr}$ in F555W and $\sim 7 \mathrm{hr}$ in F814W. The combination of the pre-explosion images for each band scaled to the planetary camera chip pixel scale $\left(0.045\right.$ pixel $\left.^{-1}\right)$, using the drizzle algorithm (Fruchter \& Hook 2002), is available from the HST Legacy Archive. ${ }^{17}$ The input images in the drizzle algorithm are weighted according to the statistical significance of each pixel, and drizzle removes the effects of geometric distortion on both image shape and photometry. In addition, it combines dithered images in the presence of cosmic rays and improves the resolution of the mosaic. Thus, the use of the drizzled image mosaics for the identification of a progenitor candidate is ideal.

Photometry of the post-explosion HST images was performed using the DOLPHOT ${ }^{18}$ package especially designed for Advanced Camera for Surveys (ACS). The post-explosion images were all obtained at the same epoch in each band and with a small dithered offset between exposures. Thus, we measured these relative offsets with respect to one fiducial image before running DOLPHOT. The output from the package automatically includes the transformation from flight-system F555W and F814W to the corresponding Johnson-Cousins (Bessell 1990) magnitudes (in $V$ and $I$ ), following the prescriptions of Sirianni et al. (2005). We include photometry of the SN in Table 2.

For the SN progenitor candidate identification, it was necessary that we use high-resolution images of the SN, ideally at the same or better resolution as the pre-SN HST image. Accordingly, we obtained HST/ACS (Wide Field Channel, $\sim 0.05$ pixel $^{-1}$ ) images in F555W and F814W on 2009 December 14, as part of our Target-of-Opportunity program GO-11575 (PI: S. Van Dyk). The SN was still quite bright at the time of these observations (see Table 2), although it was not saturated in the images. The observations in each band were in pairs of dithered $260 \mathrm{~s}$ exposures. These individual exposures were drizzled to produce final mosaics in each band. A comparison of an HST SN image to a pre-SN image is shown in Figure 7.

We achieved high-precision relative astrometry between the pre-explosion drizzled WFPC2 image in each band (the SN site is located on this chip of the pre-explosion images; this image is of the same depth as the drizzled full mosaic image) and the post-explosion ones by geometrically transforming the former to match precisely the latter. Using the IRAF task geomap, we carried out several geometric transformations between the two sets of images, using different numbers of point-like sources in common between the two data sets (from $\sim 10-30$ manually selected sources to $\gtrsim 300$ sources using an automated matching algorithm), all with rms uncertainty $\lesssim 00^{\prime \prime} 15$. We then

\footnotetext{
16 http://archive.stsci.edu/hst/.

17 http://archive.stsci.edu/hlsp/.

18 The ACS module of DOLPHOT is an adaptation of the photometry package HSTPHOT. We used v1.1, updated 2010 January 6, from http://purcell.as.arizona.edu/dolphot/.
} 

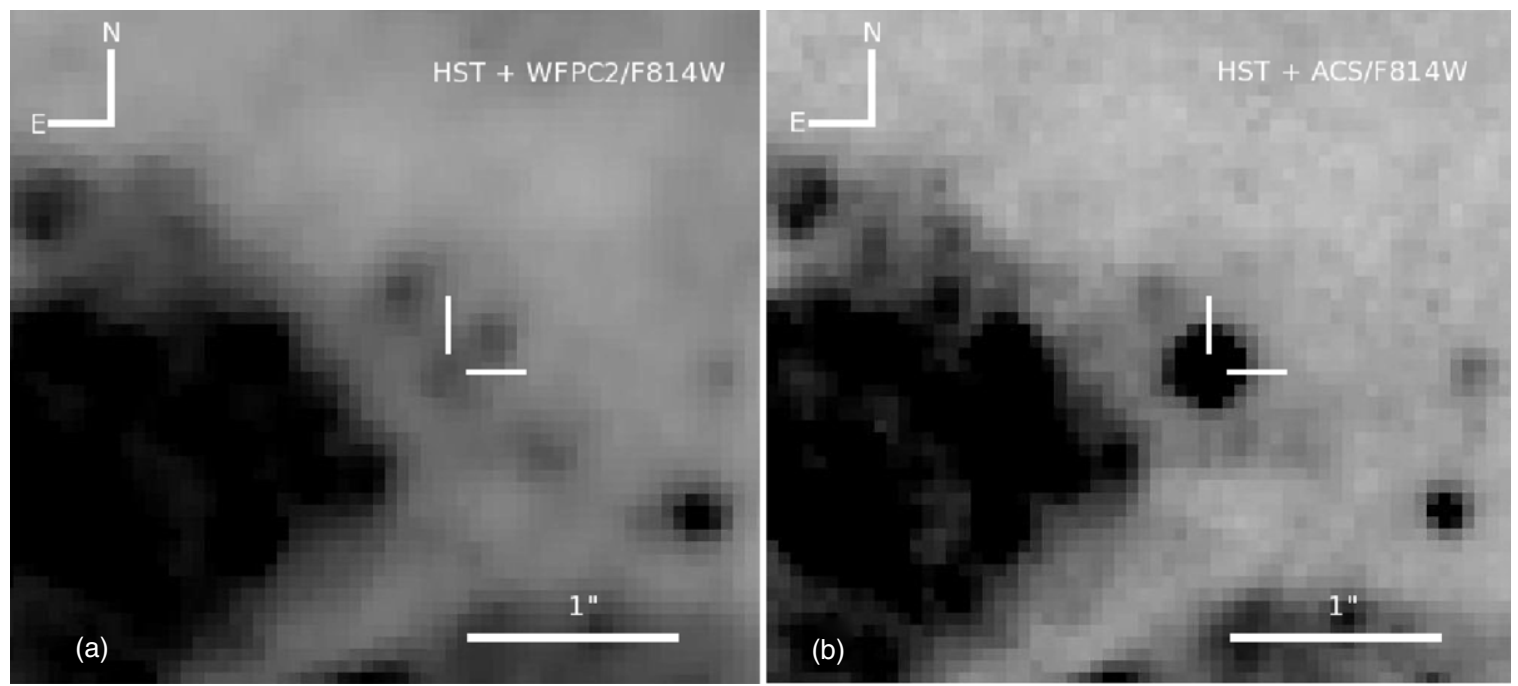

Figure 7. (a) Subsections of the pre-explosion HST WFPC2/F814W images of M66, and (b) the post-explosion HST ACS/F814W image of SN 2009hd. The positions of the candidate progenitor and the $\mathrm{SN}$ are indicated by tick marks.

transformed the pre-explosion images to the post-explosion ones using the IRAF task geotran. The positions (and their uncertainties) of the $\mathrm{SN}$ and the progenitor candidate were generally obtained by averaging the measurements from two centroiding methods, the task daofind within IRAF/DAOPHOT and imexamine within IRAF. For all solutions, the resulting SN positions in the pre-explosion images in the two bands were all consistent.

As a result, in the pre-SN F814W image we identify an object very near the SN position, which we consider to be the progenitor candidate. It is not detected at this position in the F555W image (see Figure 8). The average final pixel position in the pre-SN F814W image is [1074.82, 1634.13] for the SN, and [1074.43, 1634.10] for the candidate in the transformed SN image. The candidate is one of the detected stars near the edge of the pre-explosion error circle suggested by Maoz (2009) in his initial attempt to identify the SN progenitor.
The differences between the $\mathrm{SN}$ and the progenitor candidate positions, compared with the total estimated uncertainty in the astrometry, are given in Table 3. This latter uncertainty was calculated as a quadrature sum of the uncertainties in the SN and progenitor candidate positions, and the rms uncertainty in the geometric transformation.

From the results in Table 3, it can be seen that the difference between the $\mathrm{SN}$ position and the position of the progenitor candidate in $\mathrm{F} 814 \mathrm{~W}$ is larger in right ascension than the measurement uncertainties. This offset could be due to the proximity of one or more sources to the progenitor candidate position, which may have affected the measurement of the object's centroid, or to the difficulty in making accurate centroid measurements, given the complex background in the bright spiral arm of the host galaxy on which the progenitor candidate is located. In Figure 8, one can see that the reference object, labeled " $\mathrm{B}$ " ( $\sim 0$ ' 25 northwest of the candidate), is visible in the
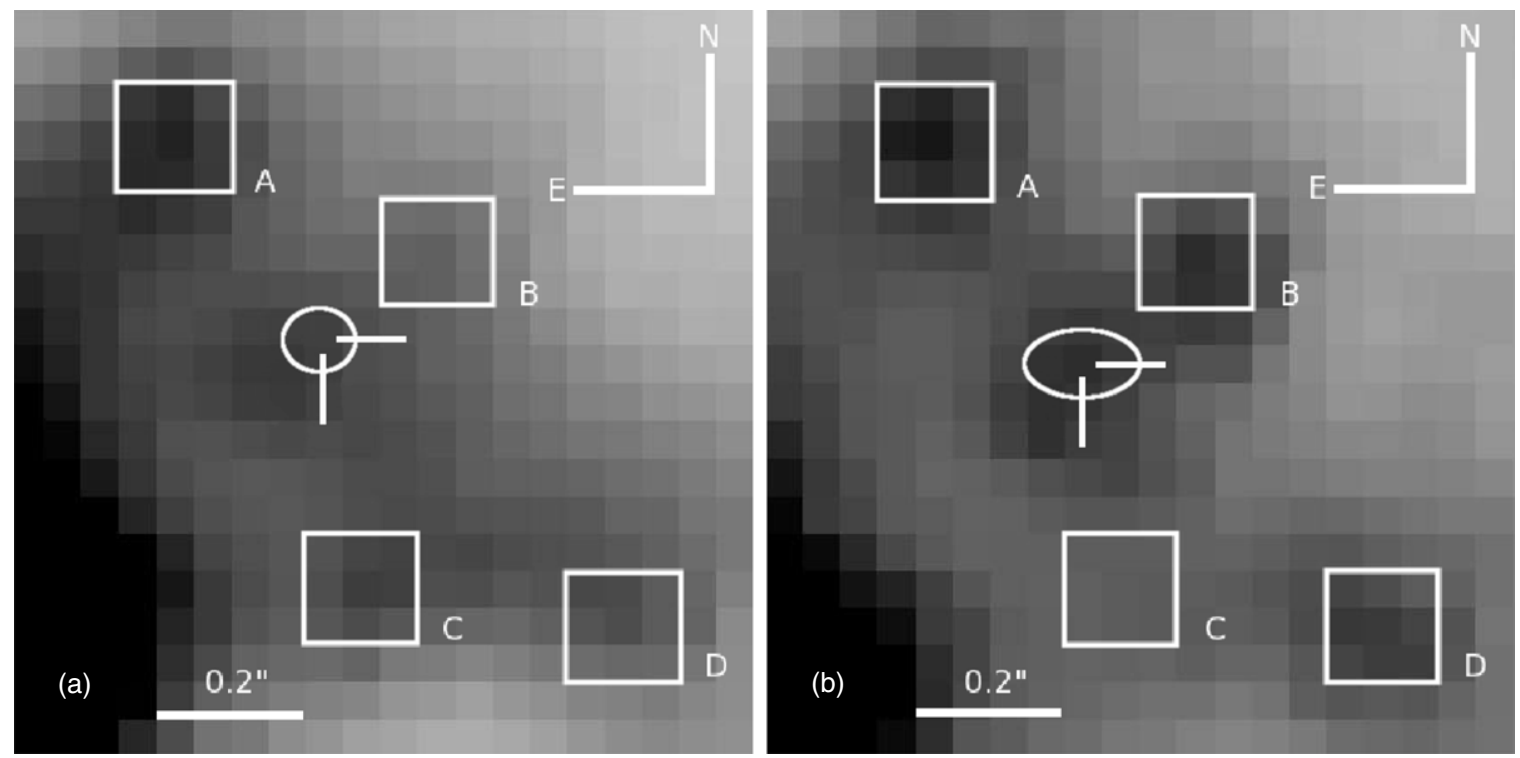

Figure 8. Subsections of the pre-explosion HST WFPC2 images of M66 in (a) F555W and (b) F814W. The position of the SN is indicated by a $5 \sigma$ positional uncertainty

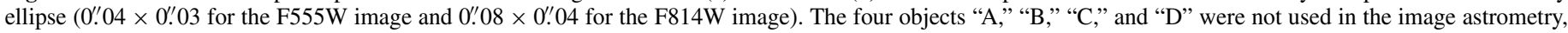
due to their relative faintness; they are labeled here only to aid the eye in locating the progenitor in the field. 
Table 3

SN and Progenitor Candidate Position Comparison

\begin{tabular}{lcr}
\hline \hline Angular Quantity & $V(\alpha / \delta)$ & $I(\alpha / \delta)$ \\
\hline Uncertainty in the progenitor position (mas) & $\ldots$ & $14 / 5$ \\
Uncertainty in the SN position (mas) & $1 / 3$ & $1 / 3$ \\
Geometric transformation (mas) & $8 / 5$ & $7 / 6$ \\
Total uncertainty (mas) & $8 / 6$ & $15 / 8$ \\
Difference in position (mas) & $\ldots$ & $20 / 1$ \\
\hline
\end{tabular}

Notes. Uncertainties in the $\mathrm{SN}$ and candidate position, in milliarcsec (mas), were estimated as the standard deviation of the average. Geometric transformation errors are derived from the positional differences between the fiducial stars used in the transformation. The total uncertainty is the quadrature sum of these uncertainties. The last line lists the residual difference between the SN and progenitor position after the geometric transformation.

F814W image, but is essentially invisible in the F555W image. The opposite is true for the object, labeled " $C$ " ( $\sim$ '.33 southwest of the candidate). We emphasize that no other point-like source can be isolated within a $1.5 \sigma$ (i.e., $\leqslant 2$ pixel) radius from the progenitor candidate position (see Figures 7 and 8). The alternative possibility is that we have not detected the progenitor for SN 2009hd. We consider both of these possibilities below.

\section{THE NATURE OF THE PROGENITOR}

Next, we attempted to measure the brightness of the progenitor candidate. We performed photometry on each of the individual pre-explosion $H S T$ images in both bands using HSTPHOT. $^{19}$ (HSTPHOT is not designed to run on the drizzled image mosaics.) We ran the package on the CR-SPLIT pairs for both bands, with option flag 10, which combines turning on the local sky determination, turning off empirically determined aperture corrections (using default values instead), and turning on PSF residual determination, with a total detection threshold of $3 \sigma$. As a result, we obtained $m_{\mathrm{F} 814 \mathrm{~W}}=23.54 \pm 0.14$ mag for the progenitor candidate. HSTPHOT did not detect in F555W the candidate detected in F814W, even with the detection threshold set as low as $1 \sigma$. To be sure that this object is truly not detected in F555W, we repeated the measurements, varying the number and order of the images for each filter input into HSTPHOT (we note that the images in each band can be split into two subsamples, depending on the various camera pointings), both automatically and forcing HSTPHOT to make a detection at the centroid position of the object in F814W. Complicating the detection is the background. The number of counts around the $\mathrm{SN}$ position in the F555W pre-SN images is nearly a factor of two higher than in a similarly sized region measured in a "empty" area, relatively free of galaxy contamination.

We note that HSTPHOT reported the progenitor candidate as having an "object type" flag of " 1 ," indicating that the source is likely stellar. Additionally, the object had a "sharpness" range of about -0.20 to 0.16 (which indicates the reliability that a detected source is indeed point-like; Dolphin 2000). A "good star" value for this parameter is between -0.3 and +0.3 (Leonard et al. 2008). Finally, the " $\chi$ " value is $\sim 1.5$; hence, it is reasonable to call the candidate a "good and clean" star $(\chi<1.5$; see Dolphin 2000). We therefore consider it very likely that the candidate is a single star.

Of course, given the offset of the SN position from this star, we must still take into account the possibility that we

\footnotetext{
19 HSTPHOT is a stellar photometry package designed for use with WFPC2
} images (Dolphin 2000). We used v1.1.7b, updated 2008 July 19.

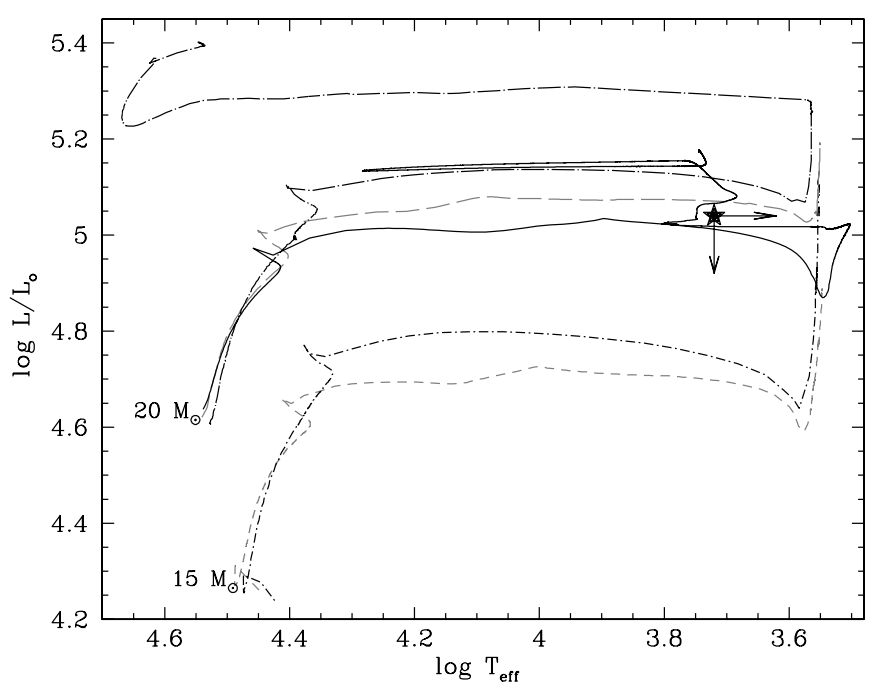

Figure 9. Hertzsprung-Russell diagram $\left(T_{\text {eff }}(\mathrm{K})\right.$ vs. $\left.L_{\text {bol }}\left(L_{\odot}\right)\right)$ showing the upper limit considering a contaminated progenitor (filled five-pointed star). Model stellar evolutionary tracks for solar metallicity are also illustrated for $M_{\text {ini }}$ of 15 and $20 M_{\odot}$ without rotation (short-dashed, and long-dashed gray lines) and with rotation of $v_{\text {ini }}=300 \mathrm{~km} \mathrm{~s}^{-1}$ (dot-short-dashed, and dot-long-dashed black lines; Hirschi et al. 2004); and for $20 M_{\odot}$ considering pulsation-induced mass-loss enhancement (solid black line; Yoon \& Cantiello 2010).

have not actually detected the progenitor in either band. We therefore attempted to constrain the brightness of an undetected progenitor by adding an artificial star at the precise SN position (see Section 3) in the pre-SN images for both bands. The PSF for the input artificial star was created from bright, isolated stars seen in the drizzled image mosaic in each band using IRAF/ DAOPHOT. The brightness of the artificial star was varied, until it was practically not detectable above the background. Then, the instrumental magnitudes of the artificial star were measured in the images using PSF fitting, and subsequently calibrated to the flight-system photometric system using the magnitude and colors of eight brighter stars in the vicinity of the SN, measured with HSTPHOT in the HST WFPC2 images. We find that $>26.1 \mathrm{mag}$ in F555W and $>23.5 \mathrm{mag}$ in F814W. Note that the limit in F814W is very similar to the measure brightness of the progenitor candidate in this band. Correcting by the same total extinction as assumed for the SN $\left(A_{V}^{\text {tot }}=3.80 \pm 0.14 \mathrm{mag}\right.$; see Section 2.3) and the adopted distance modulus $(\mu=29.86 \pm 0.08$ mag; Freedman et al. 2001), we find a limit on the absolute magnitude of an undetected progenitor, $M_{V}^{0} \gtrsim-7.6 \mathrm{mag}$. Furthermore, assuming a bolometric correction of -0.29 mag for supergiants at solar metallicity (Kurucz 1993; Kurucz ATLAS9 models, CD-ROMs 13 and 18; see below for the metallicity measurement in the SN environment) appropriate for $(V-I)_{0} \gtrsim 0.99 \mathrm{mag}$ (this is the limit on the color that arises from the reddeningcorrected upper limits at $V$ and $I$, above), we find an upper limit to such a star's bolometric luminosity of $L_{\text {bol }} \lesssim 10^{5.04} L_{\odot}$. The limit on the effective temperature, from the color limit, is then $T_{\text {eff }} \lesssim 5200 \mathrm{~K}$. We place this limit on the progenitor in the Hertzsprung-Russell (H-R) diagram in Figure 9.

We can compare the limit shown in the figure to massivestar evolutionary models without rotation and with rotation $\left(v_{\text {ini }}=300 \mathrm{~km} \mathrm{~s}^{-1}\right.$; Hirschi et al. 2004). One can see that the appropriate initial masses to consider, of those that are available for these models, are 15 and $20 M_{\odot}$. (We note that the majority of stars do not rotate as fast as those in the models used here; e.g., less than about $10 \%$ of young Galactic O-type 
stars have rotation speeds of $300 \mathrm{~km} \mathrm{~s}^{-1}$ or more; Penny et al. 2004.) We also show the evolutionary tracks for a model star of initial mass $20 M_{\odot}$ with pulsationally enhanced mass loss (Yoon \& Cantiello 2010). We assume that these tracks correspond to solar metallicity. We determined directly the metallicity in the SN environment from a neighboring $\mathrm{H}$ II region detected in the late-time SN spectrum (see Section 2.2). Measuring the $\mathrm{H} \alpha$ and $[\mathrm{NII}] \lambda 6584$ line intensities and applying a cubic-fit relation between the ratio of these two lines and the oxygen abundance (Pettini \& Pagel 2004), we find $12+\log (\mathrm{O} / \mathrm{H})=$ $8.43 \pm 0.05$, which is somewhat lower than the solar abundance, $8.69 \pm 0.05$ (Asplund et al. 2009). We adopt the criterion given by Smartt et al. (2009), who, taking into account the uncertainties in the different metallicity estimates, considers $12+\log (\mathrm{O} / \mathrm{H})=$ 8.4 as the dividing line between solar and subsolar metallicity evolutionary tracks.

To convert this progenitor detection limit to an upper limit on the initial mass of the progenitor, we considered the luminosities of the evolutionary tracks in the post-red supergiant (RSG) phase (see Figure 9). We made this estimation three different ways. First, we compared the luminosity upper limit to the stellar endpoints of the evolutionary tracks, as Fraser et al. (2010) did in the case of the SN II-L 2009kr. Doing so, we find that $M_{\text {ini }} \lesssim 20 M_{\odot}$ for the models with no rotation, and $\lesssim 15 M_{\odot}$ for the models with rotation. If we, instead, consider where the upper limit intersects the evolutionary tracks, we find a similar upper initial mass limit, $\lesssim 20 M_{\odot}$, for the non-rotating models, and $\lesssim 18 M_{\odot}$ for the models with rotation. If we consider the model with pulsationally induced mass loss at $20 \mathrm{M}_{\odot}$, one can see that it naturally achieves terminus blueward of the RSG phase. Yoon \& Cantiello (2010) found that their $17 M_{\odot}$ model only experiences pulsations after Ne burning. One can imagine, then, that, as the models approach $20 M_{\odot}$ from that lower mass, pulsations become a stronger effect, and the models also terminate toward the blue, rather than the red. Thus, conservatively, we can say that, if pulsations are important for these massive stars, the luminosity limit could correspond to a mass limit $\lesssim 20 M_{\odot}$; however, more liberally, this can extend to a somewhat lower mass limit of $\lesssim 18-19 M_{\odot}$.

\section{DISCUSSION AND CONCLUSIONS}

Observations at early times and at later (nebular) phases suggest that SN 2009hd is an SN II-L, similar to SN 1990K (Cappellaro et al. 1995) and SN 2009kr (Elias-Rosa et al. 2010). However, the lack of data between $~ 30$ and $100 \mathrm{~d}$ leaves open the possibility that SN 2009hd may be a hybrid between SNe II-L and SNe II-P. If it is an SN II-L, it is one of the most extinguished examples detected thus far, possibly as a result of proximity to a dust lane that is clearly evident in pre-SN HST images. Projection effects may further play a role, given the inclination of M66 (65 ${ }^{\circ}$; Tully 1988).

Comparing HST images of the host galaxy prior to explosion and of the $\mathrm{SN}$ at late times, the resulting high-precision relative astrometry has provided us with a means of identifying the SN progenitor. A progenitor candidate is possibly identified in the available F814W, but definitely not in the F555W, pre$\mathrm{SN}$ images. Even with the high spatial resolution of the HST WFPC2 images, the uncertainties in the astrometry are large enough that we are unable to make a definitive identification. Given the relative proximity of the host galaxy to us $(9.4 \mathrm{Mpc})$, this is rather surprising. The high extinction, combined with the complex background in the SN environment, likely leads to this level of uncertainty.
We can constrain the properties (luminosity, effective temperature) of the progenitor star for SN 2009hd. Although the inferred properties are certainly consistent with an RSG, the constraints also allow for the possibility that the star is more yellow than red. A yellow color could imply that it exploded in a post-RSG evolutionary state (or perhaps that it is an RSG in a binary system with a bluish companion). The progenitor may have evolved back to the blue after reaching the RSG stage, for example, because it has lost mass (e.g., Podsiadlowski 1992; Meynet et al. 2011) as a result of rotationally induced winds (e.g., Hirschi et al. 2004), or through pulsationally enhanced mass loss (e.g., Yoon \& Cantiello 2010). The fact that the $\mathrm{H}$ lines are weak in the nebular spectrum may indicate that the star exploded with a low-mass $\mathrm{H}$ envelope, presumably lost through high mass loss via stellar winds prior to the termination of $\mathrm{He}$ burning. Consequently, the star would evolve toward the blue before its terminus (e.g., Meynet et al. 2011 and references therein). Through comparison with high-mass stellar evolutionary tracks at solar metallicity, we are able, conservatively, to constrain the initial mass of the progenitor to $M_{\text {ini }} \lesssim 20 M_{\odot}$.

This limit to the initial mass estimate for the SN 2009hd progenitor is consistent with the range in mass found for the SN II-L $2009 \mathrm{kr}, M_{\mathrm{ini}} \approx 18-24 M_{\odot}$ (Elias-Rosa et al. 2010; see also Fraser et al. 2010). The progenitor of SN $2009 \mathrm{kr}$ was inferred to be a likely yellow supergiant. The progenitor of the unusual, high-luminosity SN II-P 2008cn also appears to have been a yellow supergiant, with $M_{\text {ini }}=15 \pm 2 M_{\odot}$ (Elias-Rosa et al. 2009). SNe 2009hd, 2009kr, and 2008cn may be a bridge between the RSGs that explode as SNe II-P and the high-mass, luminous blue variables or more extreme RSGs that may explode as SNe IIn (Smith et al. 2011).

Ultimately, a very late time set of multi-band images of the SN 2009hd field should be obtained with HST and the Wide Field Camera 3 (WFC3) many years later, when the SN has greatly faded, as has been done for the SN IIn $2005 \mathrm{gl}$ (Gal-Yam \& Leonard 2009) and for the SN IIb 1993J and SN II-P 2003gd (Maund \& Smartt 2009), to better distinguish the constituents of the SN 2009hd environment and to help decipher the true nature of its progenitor.

This research is based in part on observations made with the NASA/ESA Hubble Space Telescope, obtained from the Data Archive at the Space Telescope Science Institute, which is operated by the Association of Universities for Research in Astronomy (AURA), Inc., under NASA contract NAS 0526555; the SMARTS Consortium $1.3 \mathrm{~m}$ telescope located at Cerro Tololo Inter-American Observatory (CTIO), Chile; the $1.5 \mathrm{~m}$ telescope located at Palomar Observatory; the Liverpool Telescope operated on the island of La Palma by Liverpool John Moores University in the Spanish Observatorio del Roque de los Muchachos of the Instituto de Astrofisica de Canarias with financial support from the UK Science and Technology Facilities Council; the Nordic Optical Telescope, operated on the island of La Palma jointly by Denmark, Finland, Iceland, Norway, and Sweden, in the Spanish Observatorio del Roque de los Muchachos of the Instituto de Astrofisica de Canarias; the $6.5 \mathrm{~m}$ Magellan Clay and Baade telescopes located at Las Campanas Observatory, Chile; and the W. M. Keck Observatory, which is operated as a scientific partnership among the California Institute of Technology, the University of California, and NASA, with generous financial support from the W. M. Keck Foundation. We are grateful to the staffs 
at these observatories for their excellent assistance with the observations.

N.E.R. thanks Stefano Benetti and Alberto Noriega-Crespo for useful discussions and Avet Harutyunyan for his help. Many of the spectra used here for comparison were obtained from the Padova-Asiago Supernova Archive (ASA). This work has made use of the NASA/IPAC Extragalactic Database (NED), which is operated by the Jet Propulsion Laboratory, California Institute of Technology, under contract with NASA. This work has been supported by the MICINN grant AYA08-1839/ESP, by the ESF EUROCORES Program EuroGENESIS (MICINN grant EUI2009-04170), by SGR grants of the Generalitat de Catalunya, and by EU-FEDER funds.

The research of A.V.F.'s supernova group has been generously supported by National Science Foundation grant AST-0908886 and the TABASGO Foundation, as well as by NASA through grants AR-11248, AR-12126, and GO-11575 from the Space Telescope Science Institute, which is operated by AURA, Inc., under NASA contract NAS 5-26555.

Facilities: HST (WFPC2), HST (ACS), CTIO:1.3m (SMARTS), PO:1.5m, Hale (TripleSpec), Liverpool:2m (RATCam), NOT (ALFOSC), Magellan:Baade (IMACS), Magellan:Clay (MagE), Keck:II (DEIMOS)

\section{REFERENCES}

Asplund, M., Grevesse, N., Sauval, A. J., \& Scott, P. 2009, A\&AR, 47, 481

Barbon, R., Benetti, S., Cappellaro, E., et al. 1995, A\&AS, 110, 513

Barbon, R., Ciatti, F., \& Rosino, L. 1979, A\&A, 72, 287

Barbon, R., Ciatti, F., \& Rosino, L. 1982, A\&A, 116, 35

Baron, E., Branch, D., Hauschildt, P. H., et al. 2000, ApJ, 545, 444

Benetti, S., Cappellaro, E., Danziger, I. J., et al. 1998, MNRAS, 294, 448

Berger, E., Foley, R. J., \& Covarrubias, R. 2009, ATel, 2118, 1

Bessell, M. S. 1990, PASP, 102, 1181

Blondin, S., Prieto, J. L., Patat, F., et al. 2009, ApJ, 693, 207

Botticella, M. T., Pastorello, A., Smartt, S. J., et al. 2009, MNRAS, 398, 1041

Buta, R. J. 1982, PASP, 94, 578

Cappellaro, E., Danziger, I. J., Della Valle, M., Gouiffes, C., \& Turatto, M. 1995, A\&A, 293, 723

Cappellaro, E., Della Valle, M., Iijima, T., \& Turatto, M. 1990, A\&A, 228, 61

Cardelli, J. A., Clayton, G. C., \& Mathis, J. S. 1989, ApJ, 345, 245

Ciatti, F., \& Rosino, L. 1977, A\&A, 56, 59

Clocchiatti, A., Benetti, S., Wheeler, J. C., et al. 1996, AJ, 111, 1286

Dessart, L., \& Hillier, D. J. 2005, A\&A, 437, 667

Dolphin, A. E. 2000, PASP, 112, 1383

Elias, N., Beckman, J. E., Benetti, S., Cappellaro, E., \& Turatto, M. 2007, in Supernovae: Lights in the Darkness (http://pos.sissa.it/cgi-bin/reader/conf.cgi?confid=60)

Elias-Rosa, N. 2007, PhD thesis, Univ. La Laguna \& Univ. Padova Elias-Rosa, N., Van Dyk, S. D., Li, W., et al. 2009, ApJ, 706, 1174 Elias-Rosa, N., Van Dyk, S. D., Li, W., et al. 2010, ApJ, 714, L254 Elmhamdi, A., Danziger, I. J., Chugai, N., et al. 2003, MNRAS, 338, 939 Faber, S. M., Phillips, A. C., Kibrick, R. I., et al. 2003, Proc. SPIE, 4841, 1657 Filippenko, A. V. 1982, PASP, 94, 715

Filippenko, A. V. 1997, ARA\&A, 35, 309
Foley, R. J., Chornock, R., Filippenko, A. V., et al. 2009, AJ, 138, 376 Foley, R. J., Papenkova, M. S., Swift, B. J., et al. 2003, PASP, 115, 1220 Fraser, M., Takáts, K., Pastorello, A., et al. 2010, ApJ, 714, L280

Freedman, W. L., Madore, B. F., Gibson, B. K., et al. 2001, ApJ, 553, 47 Fruchter, A. S., \& Hook, R. N. 2002, PASP, 114, 144

Gal-Yam, A., \& Leonard, D. C. 2009, Nature, 458, 865

Hamuy, M., Pinto, P. A., Maza, J., et al. 2001, ApJ, 558, 615

Herter, T. L., Henderson, C. P., Wilson, J. C., et al. 2008, Proc. SPIE, 7014, 30

Hirschi, R., Meynet, G., \& Maeder, A. 2004, A\&A, 425, 649

Horne, K. 1986, PASP, 98, 609

Kasliwal, M. M. 2009, ATel, 2113, 1

Kasliwal, M. M., Sahu, D. K., \& Anupama, G. C. 2009, CBET, 1874, 1

Kelson, D. D. 2003, PASP, 115, 688

Kennicutt, R. C., Jr., Armus, L., Bendo, G., et al. 2003, PASP, 115, 928

Kurucz, R. L. 1993, ATLAS9 CDROMs 13 and 18

Leonard, D. C., Filippenko, A. V., Gates, E. L., et al. 2002, PASP, 114, 35

Leonard, D. C., Gal-Yam, A., Fox, D. B., et al. 2008, PASP, 120, 1259

Li, W., Leaman, J., Chornock, R., et al. 2011, MNRAS, 412, 1441

Maoz, D. 2009, ATel, 2114, 1

Marshall, J. L., Burles, S., Thompson, I. B., et al. 2008, Proc. SPIE, 7014, 169 Matthews, K., Neugebauer, G., Armus, L., \& Soifer, B. T. 2002, AJ, 123, 753 Maund, J. R., \& Smartt, S. J. 2009, Science, 324, 486

Meynet, G., Georgy, C., Hirschi, R., et al. 2011, Bull. Soc. R. Sci. Liege, 80, 266

Monard, L. A. G. 2009, CBET, 1867, 1

Olivares, E. F., Hamuy, M., Pignata, G., et al. 2010, ApJ, 715, 833

Pastorello, A., Sauer, D., Taubenberger, S., et al. 2006, MNRAS, 370, 1752

Patat, F., Barbon, R., Cappellaro, E., \& Turatto, M. 1994, A\&AS, 98, 443

Penny, L. R., Sprague, A. J., Seago, G., \& Gies, D. R. 2004, ApJ, 617, 1316

Pettini, M., \& Pagel, B. E. J. 2004, MNRAS, 348, 59

Podsiadlowski, P. 1992, PASP, 104, 717

Poznanski, D., Butler, N., Filippenko, A. V., et al. 2009, ApJ, 694, 1067

Poznanski, D., Ganeshaligam, M., Silverman, J. M., \& Filippenko, A. V. 2011, MNRAS, 415, L81

Pozzo, M., Meikle, W. P. S., Rayner, J. T., et al. 2006, MNRAS, 368, 1169

Prieto, J. L., Kistler, M. D., Thompson, T. A., et al. 2008, ApJ, 681, L9

Saha, A., Sandage, A., Tammann, G. A., et al. 1999, ApJ, 522, 802

Schlegel, D. J., Finkbeiner, D. P., \& Davis, M. 1998, ApJ, 500, 525

Schlegel, E. M. 1996, AJ, 111, 1660

Sirianni, M., Jee, M. J., Bentez, N., et al. 2005, PASP, 117, 1049

Smartt, S. J., Eldridge, J. J., Crockett, R. M., \& Maund, J. R. 2009, MNRAS, 395, 1409

Smith, N., Ganeshalingam, M., Chornock, R., et al. 2009, ApJ, 697, L49

Smith, N., Li, W., Filippenko, A. V., \& Chornock, R. 2011, MNRAS, 412, 1522

Spitzer, L., Jr. 1948, ApJ, 108, 276

Stetson, P. B. 1987, PASP, 99, 191

Stritzinger, M., Burns, C. R., Phillips, M. M., et al. 2011, AJ, 140, 2036

Swartz, D. A., Wheeler, J. C., \& Harkness, R. P. 1991, ApJ, 374, 266

Taubenberger, S., Navasardyan, H., Maurer, J. I., et al. 2011, MNRAS, 413, 2140

Tsvetkov, D. Y. 1983, Perem. Zvezdy, 22, 39

Tully, R. B. 1988, Nearby Galaxies Catalog (Cambridge: Cambridge Univ. Press)

Vacca, W. D., Cushing, M. C., \& Rayner, J. T. 2003, PASP, 115, 389

Van Dyk, S. D., Peng, C. Y., King, J. Y., et al. 2000, PASP, 112, 1532

Wade, R. A., \& Horne, K. 1988, ApJ, 324, 411

Weiner, B. J., Phillips, A. C., Faber, S. M., et al. 2005, ApJ, 620, 595

Wells, L. A., Phillips, M. M., Suntzeff, B., et al. 1994, AJ, 108, 2233

Yamaoka, H., \& Itagaki, K. 2009, CBET, 1874, 1

Yoon, S.-C., \& Cantiello, M. 2010, ApJ, 717, L62 